\title{
THE STRUCTURE OF SOLUTIONS FOR EQUATIONS RELATED TO THE MOTIONS OF PLANE CURVES
}

\author{
JONG-SHENQ GUO ${ }^{1}$, CHU-PIN LO ${ }^{2}$ and JE-CHIANG TSAI ${ }^{1}$
}

(Received 22 May, 2002; revised 29 August, 2002)

\begin{abstract}
We study the structure of solutions of an initial value problem arising in the study of steadily rotating spiral waves in the kinematic theory of excitable media. In particular, we prove that under certain conditions there is a unique global positive monotone increasing solution.
\end{abstract}

\section{Introduction}

In this paper, we study the initial value problem $(\mathrm{P})$ :

$$
\begin{aligned}
& w^{\prime \prime}-a(w) w^{\prime}+\eta=0, \quad y>0, \\
& w(0)=0, \quad w^{\prime}(0)=1,
\end{aligned}
$$

where $\eta \in \mathbb{R}$ is a parameter and $a(s)$ is a piecewise $C^{1}$ function satisfying $a(0)=0$, $a^{\prime}(s)>0$ for $s \neq 0, a(s) \rightarrow-\infty$ as $s \rightarrow-\infty$, and

$$
\int_{0}^{\infty} \frac{d s}{1+A(s)}<\infty
$$

where $A(s):=\int_{0}^{s} a(t) d t$. Note that $A(s) \rightarrow \infty$ as $s \rightarrow-\infty$. A typical example is

$$
a(s)=|s|^{\alpha-1} s, \quad \alpha>0 .
$$

In particular, when $a(s)=s$ the initial value problem $(\mathrm{P})$ arises in the study of steadily rotating spiral waves in the kinematic theory of excitable media. See, for

\footnotetext{
'Department of Mathematics, National Taiwan Normal University, 88, S-4 Ting Chou Road, Taipei 117 , Taiwan.

${ }^{2}$ Department of Finance and Taxation, Dahan Institute of Technology, I, Sujen Street, Dahan, Sincheng, Hualian 971, Taiwan.

(C) Australian Mathematical Society 2004, Serial-fee code 1446-1811/04
} 
example, [1, 4, 5], especially the nice review article of Meron [4] in 1992 and the references cited therein. Let $\kappa$ be the curvature of a steadily rotating spiral wave whose tip neither grows nor retracts, $\kappa_{c}$ be the curvature of the spiral arm at the tip, $c_{r}$ be the normal velocity, $s$ be the arc length, and $\omega$ be the constant frequency. Then we have the following relation (see, for example, (4.48) and (4.49) in [4]):

$$
\begin{gathered}
\kappa\left(\int_{0}^{s} \kappa(\xi) c_{r}(\xi) d \xi\right)+\frac{d c_{r}}{d s}=\omega, \quad s>0, \\
\lim _{s \rightarrow 0} \kappa(s)=\kappa_{c}, \quad \lim _{s \rightarrow \infty} \kappa(s)=0 .
\end{gathered}
$$

Using the following curvature-speed relation $c_{r}=c-D \kappa$, where $c$ and $D$ are positive constants, and by neglecting the higher order term of $\kappa$ in the integral of (1.4), we have

$$
c \kappa\left(\int_{0}^{s} \kappa(\xi) d \xi\right)-D \frac{d \kappa}{d s}=\omega
$$

Set

$$
q(s)=\int_{0}^{s} \kappa(\xi) d \xi
$$

Then $q^{\prime}(s)=\kappa(s)$ and $q$ satisfies

$$
D q^{\prime \prime}-c q q^{\prime}+\omega=0, \quad s>0 .
$$

Now we introduce the change of variables:

$$
y=\sqrt{\frac{c \kappa_{c}}{D}} s, \quad w(y)=\sqrt{\frac{c}{D \kappa_{c}}} q(s), \quad \eta=\frac{\omega}{\sqrt{c D \kappa_{c}^{3}}} .
$$

Then $w(y)$ satisfies (1.1) with $a(s)=s$. Also $w(0)=0, w^{\prime}(0)=1$ and $w^{\prime}(y) \rightarrow 0$ as $y \rightarrow \infty$.

For more references on spiral waves, we refer the reader to the paper of Ikota, Ishimura and Yamaguchi [3], the recent paper of Giga, Ishimura and Kohsaka [2], and the references cited therein.

The local existence and uniqueness of solutions of $(\mathrm{P})$ for any given $\eta \in \mathbb{R}$ is trivial. We denote this solution by $w(y ; \eta)$. Note that $w>0$ and $w^{\prime}>0$ for small $y>0$.

The main theorem of this paper is as follows.

THEOREM 1.1. There is a unique positive constant $\eta^{*}$ such that the following hold:

(i) if $\eta>\eta^{*}$, then $w(y ; \eta)$ has exactly one critical point and $w$ exists globally such that $w(y ; \eta) \rightarrow-\infty$ and $w^{\prime}(y ; \eta) \rightarrow 0$ as $y \rightarrow \infty$ 
(ii) if $\eta=\eta^{*}$, then $w^{*}(y):=w\left(y ; \eta^{*}\right)$ is a global positive monotone increasing solution such that $w^{*}(y) \rightarrow \infty$ and $\left(w^{*}\right)^{\prime}(y) \rightarrow 0$ as $y \rightarrow \infty$;

(iii) if $\eta<\eta^{*}$, then $w(y ; \eta)$ is monotone increasing in $[0, R)$ such that $w(y ; \eta) \rightarrow$ $\infty$ and $w^{\prime}(y ; \eta) \rightarrow \infty$ as $y \rightarrow R^{-}$for some $R<\infty$.

We give an interpretation of Theorem 1.1 for the case when $a(s)=s$ as follows. In this case, we have found a family of steadily rotating spiral waves. In this family there is a unique frequency $\omega^{*}:=\eta^{*} \sqrt{c D \kappa_{c}^{3}}$ such that any curve in the plane with this frequency is a spiral wave with positive curvature. If we increase the frequency $\omega$ so that $\omega>\omega^{*}$, then the curvature of the curve would change sign exactly once. Furthermore, it follows from (1.5) that the rotation number defined by

$$
\frac{1}{2 \pi} \int_{0}^{\infty} \kappa(s) d s
$$

is $+\infty$ when $\omega=\omega^{*}$ and is $-\infty$ when $\omega>\omega^{*}$. Hence they are indeed spiral waves.

This paper is organised as follows. In Section 2, we study the properties of various solutions of $(\mathrm{P})$ for different values of the parameter $\eta$. After deriving a comparison principle, we prove Theorem 1.1 in Section 3. In particular, there is a unique global positive monotone increasing solution of (P). Finally, we give a discussion in Section 4.

\section{Structure of solutions}

We first note that, by (1.3), $A(s) \rightarrow \infty$ as $s \rightarrow \infty$. Moreover, $a(s) \rightarrow \infty$ as $s \rightarrow \infty$. Otherwise $A(s)$ grows at most linearly and so the first integral in (1.3) diverges.

Let $w(y ; \eta)$ be the solution of $(\mathrm{P})$ for a given $\eta \in \mathbb{R}$. Let

$$
F(y)=w^{\prime}(y)-A(w(y)), \quad y \geq 0 .
$$

By (1.1) and (1.2), it is easy to see that $F^{\prime}(y)=-\eta$ and $F(0)=1$. Hence we obtain that

$$
w^{\prime}(y)-A(w(y))=1-\eta y .
$$

We shall see that the above relation plays an important role in this study.

LEMMA 2.1. Suppose that $\eta \leq 0$. Then $w(y ; \eta)$ is monotone increasing in $[0, R)$ such that $w(y ; \eta) \rightarrow \infty$ and $w^{\prime}(y ; \eta) \rightarrow \infty$ as $y \rightarrow R^{-}$for some $R<\infty$.

PROOF. Let $w(y)=w(y ; \eta)$. By assumption, we have

$$
w^{\prime}(y) \geq A(w(y))+1>0
$$


for $y \geq 0$. Hence $w>0$ in its existence interval $[0, R)$. have

We claim that $R<\infty$. For contradiction, we suppose that $R=\infty$. By (2.3), we and so

$$
\frac{w^{\prime}(y)}{1+A(w(y))} \geq 1, \quad \forall y \geq 0
$$

$$
\int_{0}^{w(y)} \frac{d s}{1+A(s)} \geq y \rightarrow \infty
$$

as $y \rightarrow \infty$, contradicting (1.3). Hence $R<\infty$. It is trivial that $w(y) \rightarrow \infty$ and $w^{\prime}(y) \rightarrow \infty$ as $y \rightarrow R^{-}$. This completes the proof.

In what follows, we always assume that $\eta>0$. Since $w^{\prime \prime}<0$ for any critical point of $w$, there is at most one critical point of $w$.

LEMMA 2.2. Suppose that for some $\eta>0$ the corresponding solution $w(y ; \eta)$ has exactly one critical point. Then $w$ is global such that $w(y ; \eta) \rightarrow-\infty$ and $w^{\prime}(y ; \eta) \rightarrow 0$ as $y \rightarrow \infty$.

PROOF. Note that by assumption there is a unique $y_{0}>0$ such that $w^{\prime}\left(y_{0}\right)=0$. Then $w^{\prime}>0$ in $\left[0, y_{0}\right)$ and $w^{\prime}<0$ for $y>y_{0}$ as long as $w$ exists.

Suppose that $w$ is not global. Then $w(y) \rightarrow-\infty$ as $y \rightarrow R^{-}$for some $R<\infty$. Otherwise, if $\lim _{y \rightarrow R^{-}} w(y)$ is finite, then there is a sequence $\left\{y_{n}\right\}$ such that $y_{n} \rightarrow R$ and $w^{\prime}\left(y_{n}\right) \rightarrow 0$ as $n \rightarrow \infty$. By (2.2), the $\operatorname{limit}_{\lim _{y \rightarrow R^{-}}} w^{\prime}(y)$ exists. Then we have $w^{\prime}(y) \rightarrow 0$ as $y \rightarrow R^{-}$. This implies that $w$ can be continued beyond $R$, a contradiction. Hence $w(y) \rightarrow-\infty$ as $y \rightarrow R^{-}$. But from (2.2) again it follows that $w^{\prime}(y)=A(w(y))+1-\eta y \rightarrow+\infty$ as $y \rightarrow R^{-}$, another contradiction. Hence $w$ is global.

Note that the limit $l:=\lim _{y \rightarrow \infty} w(y)$ exists. If $l>-\infty$, then, by (2.2), $\lim _{y \rightarrow \infty} w^{\prime}(y)=-\infty$. This is impossible. Hence $w(y) \rightarrow-\infty$ as $y \rightarrow \infty$.

Next, we claim that either $w^{\prime \prime}<0$ in $[0, \infty)$ or $w^{\prime \prime}>0$ in $\left(y_{1}, \infty\right)$ for some $y_{1}>0$ with $w^{\prime \prime}\left(y_{1}\right)=0$. Indeed, if there exists $y_{1}>0$ such that $w^{\prime \prime}<0$ in $\left[0, y_{1}\right)$ and $w^{\prime \prime}\left(y_{1}\right)=0$, then, by $(1.1), w\left(y_{1}\right) \neq 0$ and $w^{\prime}\left(y_{1}\right) \neq 0$. By differentiating (1.1) with respect to $y$, we obtain that

$$
w^{\prime \prime \prime}=a(w) w^{\prime \prime}+a^{\prime}(w)\left(w^{\prime}\right)^{2} .
$$

Hence $w^{\prime \prime \prime}\left(y_{1}\right)>0$ and so $w^{\prime \prime}(y)>0$ for $y \in\left(y_{1}, y_{1}+\delta\right)$ for some $\delta>0$. It follows from (2.4) and the fact that $a^{\prime}(s)>0, \forall s \neq 0$, that $w^{\prime \prime \prime}>0$ when $w^{\prime \prime}=0$. Therefore $w^{\prime \prime}>0$ in $\left(y_{1}, \infty\right)$.

In any case, the limit $L:=\lim _{y \rightarrow \infty} w^{\prime}(y)$ exists and $L \leq 0$. If $L<0$, then, by $(1.1), w^{\prime \prime}(y) \rightarrow \infty$ as $y \rightarrow \infty$. This contradicts the assumption $w^{\prime}<0$ in $\left(y_{0}, \infty\right)$. We conclude that $w^{\prime}(y) \rightarrow 0$ as $y \rightarrow \infty$. This proves the lemma. 
From now on we assume that $w^{\prime}>0$ in its existence interval. By differentiating (1.1) with respect to $y$, we obtain for $v(y):=w^{\prime}(y)$ that

$$
v^{\prime \prime}=a(w) v^{\prime}+a^{\prime}(w) v^{2}, \quad v(0)=1
$$

Hence by (2.5) the function $v$ has at most one critical point. Note that $v^{\prime}(0)=w^{\prime \prime}(0)=$ $-\eta<0$.

LEMMA 2.3. Given $\eta>0$, suppose that $w^{\prime}(y ; \eta)>0$ in its existence interval and that $w^{\prime}(y ; \eta)$ has exactly one critical point. Then $w(y ; \eta) \rightarrow \infty$ and $w^{\prime}(y ; \eta) \rightarrow \infty$ as $y \rightarrow R^{-}$for some $R<\infty$.

Proof. Suppose that $w$ is global. We claim that $w(y) \rightarrow \infty$ and $w^{\prime}(y) \rightarrow \infty$ as $y \rightarrow \infty$. Since $w^{\prime}>0$, the limit $l:=\lim _{y \rightarrow \infty} w(y)$ exists and $l>0$. By (2.2), $A(w(y))=w^{\prime}(y)+\eta y-1 \geq \eta y-1 \rightarrow \infty$ as $y \rightarrow \infty$. Hence $l=\infty$. Suppose that $v^{\prime}\left(y_{0}\right)=0$ for some $y_{0}>0$. Then by assumption $v^{\prime}>0$ for $y>y_{0}$. Hence the limit $L:=\lim _{y \rightarrow \infty} w^{\prime}(y)$ exists and $L>0$. If $L<\infty$, then, by $(1.1), w^{\prime \prime}(y) \rightarrow \infty$ as $y \rightarrow \infty$, a contradiction. Therefore $L=\infty$. It follows from l'Hôpital's rule that

$$
\lim _{y \rightarrow \infty} \frac{y}{1+A(w(y))}=\lim _{y \rightarrow \infty} \frac{1}{a(w(y)) w^{\prime}(y)}=0 .
$$

Now, from (2.2) and (2.6) it follows that

$$
\lim _{y \rightarrow \infty} \frac{w^{\prime}(y)}{1+A(w(y))}=1-\eta \lim _{y \rightarrow \infty} \frac{y}{1+A(w(y))}=1 .
$$

Thus there exists $y_{1}>y_{0}$ such that

$$
\frac{w^{\prime}(y)}{1+A(w(y))} \geq \frac{1}{2}, \quad \forall y \geq y_{1} .
$$

By integrating (2.7) from $y_{1}$ to $y$ and letting $y \rightarrow \infty$, this leads to a contradiction. Hence $w$ cannot be global.

It is clear that $w(y ; \eta) \rightarrow \infty$ and $w^{\prime}(y ; \eta) \rightarrow \infty$ as $y \rightarrow R^{-}$for some $R<\infty$. The lemma follows.

The following lemma is about the case when $v$ has no critical point.

LEMMA 2.4. Suppose that $w(y ; \eta)$ is a global solution such that $w^{\prime}(y ; \eta)>0$ for all $y \in(0, \infty)$ for some $\eta>0$. Then $w^{\prime \prime}<0$ in $(0, \infty), w(y ; \eta) \rightarrow \infty$ and $w^{\prime}(y ; \eta) \rightarrow 0$ as $y \rightarrow \infty$. Conversely, if $w^{\prime}(y ; \eta)>0$ and $w^{\prime \prime}(y ; \eta)<0$ as long as $w$ exists for some $\eta>0$, then $w(y ; \eta)$ is global. 
Proof. From $A(w(y))=w^{\prime}(y)+\eta y-1$, it follows that $w(y) \rightarrow \infty$ as $y \rightarrow \infty$. Since $w$ is global, by Lemma $2.3, w^{\prime \prime}<0$ in $(0, \infty)$. For the contradiction, suppose that the limit $l:=\lim _{y \rightarrow \infty} w^{\prime}(y)>0$. Then, by (1.1), $w^{\prime \prime}(y) \rightarrow \infty$ as $y \rightarrow \infty$, a contradiction. Hence $w^{\prime}(y ; \eta) \rightarrow 0$ as $y \rightarrow \infty$.

Now, suppose that there is an $\eta>0$ such that $w^{\prime}(y ; \eta)>0$ and $w^{\prime \prime}(y ; \eta)<0$ in the existence interval and that $w(y ; \eta)$ is not global. Then $w(y) \rightarrow \infty$ as $y \rightarrow R^{-}$for some $R<\infty$. Otherwise, by (2.2), both $w(y)$ and $w^{\prime}(y)$ have finite limits and then $w$ can be continued beyond $R$, a contradiction. Hence $w(y) \rightarrow \infty$ as $y \rightarrow R^{-}$for some $R<\infty$. Therefore, by (2.2) again, $w^{\prime}(y) \rightarrow \infty$ as $y \rightarrow R^{-}$, which contradicts the assumption that $w^{\prime \prime}<0$. Hence $w(y ; \eta)$ is global if $w^{\prime}(y ; \eta)>0$ and $w^{\prime \prime}(y ; \eta)<0$ as long as $w$ exists. This proves the lemma.

\section{Proof of Theorem 1.1}

For convenience, we denote the existence interval of $w(y ; \eta)$ by $[0, R)$ for some $R=R(\eta)>0$. We begin with the following comparison principle.

LEMMA 3.1. Let $w_{i}(y)=w\left(y ; \eta_{i}\right), R_{i}=R\left(\eta_{i}\right), i=1,2$, and $R=\min \left\{R_{1}, R_{2}\right\}$. Suppose that $w_{2}^{\prime}>0$ in $\left[0, R_{2}\right)$ and $\eta_{1}<\eta_{2}$. Then $w_{1}^{\prime}>w_{2}^{\prime}$ in $(0, R)$ and $w_{1}^{\prime \prime}>w_{2}^{\prime \prime}$ in $[0, R)$.

Proof. Note that, by (1.1), the lemma holds for $y$ small, since $\eta_{1}<\eta_{2}$. Let $v_{i}=w_{i}^{\prime}$. It follows from (1.1) that

$$
\begin{aligned}
\left(v_{1}-v_{2}\right)^{\prime} & =a\left(w_{1}\right) v_{1}-a\left(w_{2}\right) v_{2}+\left(\eta_{2}-\eta_{1}\right) \\
& =a\left(w_{1}\right)\left(v_{1}-v_{2}\right)+\left[a\left(w_{1}\right)-a\left(w_{2}\right)\right] v_{2}+\left(\eta_{2}-\eta_{1}\right)
\end{aligned}
$$

Therefore to prove the lemma it suffices to prove that $w_{1}^{\prime}>w_{2}^{\prime}$ in $(0, R)$.

For the contradiction, suppose that there is $y_{0} \in(0, R)$ such that $w_{1}^{\prime}>w_{2}^{\prime}$ in $\left(0, y_{0}\right)$ and $w_{1}^{\prime}\left(y_{0}\right)=w_{2}^{\prime}\left(y_{0}\right)$. Then $w_{1}\left(y_{0}\right)>w_{2}\left(y_{0}\right)>0$ and $v_{1}^{\prime}\left(y_{0}\right) \leq v_{2}^{\prime}\left(y_{0}\right)$, contradicting (3.1). This completes the proof.

The following lemma gives the uniqueness of a global monotone increasing solution of $(\mathrm{P})$.

LEMMA 3.2. There is at most one global monotone increasing solution of (P) such that $w(y) \rightarrow \infty$ and $w^{\prime}(y) \rightarrow 0$ as $y \rightarrow \infty$.

PROOF. Suppose that there are two distinct global monotone increasing solutions $w_{1}, w_{2}$ of $(\mathrm{P})$ such that $w_{i}(y) \rightarrow \infty$ and $w_{i}^{\prime}(y) \rightarrow 0$ as $y \rightarrow \infty, i=1,2$. Suppose that 
$\eta_{1}<\eta_{2}$, where $\eta_{i}=-w_{i}^{\prime \prime}(0), i=1,2$. Then, by Lemma 3.1, $\left[w_{1}^{\prime}(y)-w_{2}^{\prime}(y)\right]^{\prime}>0$ in $[0, \infty)$. Since $w_{1}^{\prime}(1)-w_{2}^{\prime}(1)>0$, the $\operatorname{limit}_{\lim } \rightarrow \infty\left[w_{1}^{\prime}(y)-w_{2}^{\prime}(y)\right]$ is strictly bigger than zero, a contradiction. The lemma follows.

We introduce the following two sets:

$$
\begin{aligned}
& I_{1}=\left\{\eta>0 \mid w(y ; \eta) \rightarrow \infty \text { and } w^{\prime}(y ; \eta) \rightarrow \infty \text { as } y \rightarrow R^{-} \text {for some } R<\infty\right\}, \\
& I_{2}=\{\eta>0 \mid w(y ; \eta) \text { has exactly one critical point }\} .
\end{aligned}
$$

By the continuous dependence on the parameter $\eta$ in (1.1), it is trivial that the set $I_{2}$ is open.

LEMMA 3.3. The set $I_{2}$ is nonempty.

PROOF. Suppose that $I_{2}=\emptyset$. Then $w^{\prime}(y ; \eta)>0, \forall y \geq 0$, for all $\eta>0$. We claim that $R(\eta) \geq R(0)$ for all $\eta>0$. From (2.2) it follows that

$$
\frac{w^{\prime}(y)}{1+A(w(y))} \leq 1, \quad \forall y \in[0, R(\eta)) .
$$

By an integration of (3.2), we get $w(y) \leq u(y)$ as long as $w$ and $u$ exist, where $u(y)$ is the solution of (1.1) with $\eta=0$. Note that $u^{\prime}>0$ in $\left[0, R_{0}\right)$ and $u(y) \rightarrow \infty$ as $y \rightarrow R_{0}^{-}$for some $R_{0}:=R(0) \in(0, \infty)$. Hence $R(\eta) \geq R_{0}$ for all $\eta>0$. Notice that $\left\{w\left(R_{0} / 2 ; \eta\right)\right\}_{\eta>0}$ is uniformly bounded.

On the other hand, from (2.2) it follows that

$$
w^{\prime}\left(R_{0} / 2 ; \eta\right)=A\left(w\left(R_{0} / 2 ; \eta\right)\right)+1-\eta R_{0} / 2 \rightarrow-\infty
$$

as $\eta \rightarrow \infty$, a contradiction. Thus $I_{2}$ is nonempty and the proof is complete.

We observe that for any $\eta \in(0, \infty) \backslash I_{2}$ the solution $w$ satisfies $w^{\prime}(y)>0$ in $[0, R(\eta))$. Hence $I_{2}$ is connected by the comparison principle (Lemma 3.1). Therefore $I_{2}=\left(\eta^{*}, \infty\right)$ for some $\eta^{*} \geq 0$. Indeed, $\eta^{*}=\inf I_{2}$.

By Lemmas 2.3 and 2.4 , the solution $v(y ; \eta)$ of $(2.5)$ has one critical point if and only if $\eta \in I_{1}$. By the continuous dependence on the parameter $\eta$ in (2.5), the set $I_{1}$ is open. Hence $\eta^{*} \notin I_{1} \cup I_{2}$.

Let $w^{*}$ be the corresponding solution of (1.1) for $\eta=\eta^{*}$. Then $\left(w^{*}\right)^{\prime}>0$ as long as $w^{*}$ exists. Moreover, $w^{*}$ is global. Otherwise, if $R\left(\eta^{*}\right)<\infty$, then $w^{*}(y) \rightarrow \infty$ as $y \rightarrow R^{-}\left(\eta^{*}\right)$. Hence $\left(w^{*}\right)^{\prime}(y) \rightarrow \infty$ as $y \rightarrow R^{-}\left(\eta^{*}\right)$, by (2.2). This implies that $\eta^{*} \in I_{1}$, a contradiction. Hence $w^{*}$ is global. Also, it follows from Lemma 2.4 that $w^{*}(y) \rightarrow \infty$ and $\left(w^{*}\right)^{\prime}(y) \rightarrow 0$ as $y \rightarrow \infty$. We conclude from Lemma 2.1 that $\eta^{*}>0$.

Observe that any solution $w(y ; \eta)$ of $(\mathrm{P})$ with $\eta \notin I_{1} \cup I_{2}$ is global and satisfies $w(y) \rightarrow \infty$ and $w^{\prime}(y) \rightarrow 0$ as $y \rightarrow \infty$. Hence $I_{1}=\left(0, \eta^{*}\right)$. This completes the proof of Theorem 1.1. 


\section{Discussion}

In this paper, we first reduce the initial value problem $(\mathrm{P})$ for a second-order ODE to a first-order ODE (2.2) by an integration. It is trivial that the problem (P) is equivalent to (2.2) with the initial value $w(0)=0$. Using this important relationship, we are able to classify the structure of solutions of $(P)$. Then in Theorem 1.1 we prove the characterisation of solutions of $(\mathrm{P})$ with respect to the parameter $\eta$.

In the case of excitable media, that is, when $a(s)=s$, we have found a family of steadily rotating spiral waves. In this family there is a unique frequency $\omega^{*}$ such that any curve in the plane with this frequency is a spiral wave with positive curvature. If we increase the frequency $\omega$ so that $\omega>\omega^{*}$, then the curvature of the wave would change sign exactly once.

\section{Acknowledgements}

This work was partially supported by the National Science Council of the Republic of China under the contract NSC 90-2115-M-003-009. We would like to thank the referee for some helpful comments on the presentation of this paper.

\section{References}

[1] P. K. Brazhnik, "Exact solutions for the kinematic model of autowaves in two-dimensional excitable media", Physica D 94 (1996) 205-220.

[2] Y. Giga, N. Ishimura and Y. Kohsaka, "Spiral solutions for a weakly anisotropic curvature flow equation", Adv. Math. Sci. Appl. 12 (2002) 393-408.

[3] R. Ikota, N. Ishimura and T. Yamaguchi, "On the structure of steady solutions for the kinematic model of spiral waves in excitable media", Japan J. Indust. Appl. Math. 15 (1998) 317-330.

[4] E. Meron, "Pattern formation in excitable media", Phys. Reports (Review Section of Phys. Lett.) 218 (1992) 1-66.

[5] A. S. Mikhailov, V. A. Davydov and A. S. Zykov, "Complex dynamics of spiral waves and motion of curves", Physica D 70 (1994) 1-39. 